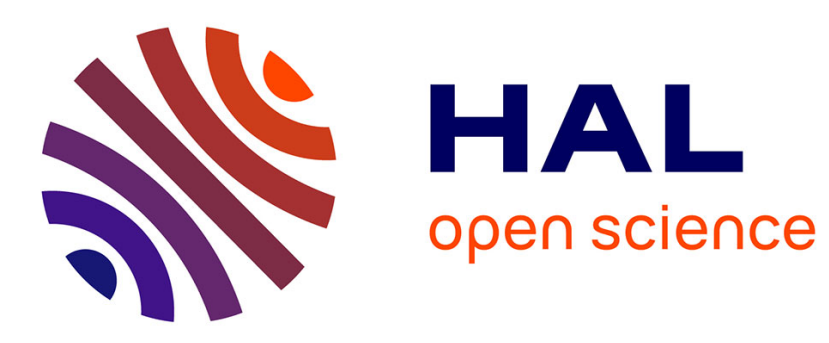

\title{
UMPa: A Multi-objective, multi-level partitioner for communication minimization
}

\author{
Umit V. Catalyurek, Mehmet Deveci, Kamer Kaya, Bora Uçar
}

\section{To cite this version:}

Umit V. Catalyurek, Mehmet Deveci, Kamer Kaya, Bora Uçar. UMPa: A Multi-objective, multilevel partitioner for communication minimization. David A. Bader; Henning Meyerhenke; Peters Sanders; Dorothea Wagner. Graph Partitioning and Graph Clustering 2012, 588, AMS, pp.53-66, 2013, Contemporary Mathematics, 10.1090/conm/588/11704 . hal-00763563

\section{HAL Id: hal-00763563 https://hal.inria.fr/hal-00763563}

Submitted on 19 Dec 2019

HAL is a multi-disciplinary open access archive for the deposit and dissemination of scientific research documents, whether they are published or not. The documents may come from teaching and research institutions in France or abroad, or from public or private research centers.
L'archive ouverte pluridisciplinaire HAL, est destinée au dépôt et à la diffusion de documents scientifiques de niveau recherche, publiés ou non, émanant des établissements d'enseignement et de recherche français ou étrangers, des laboratoires publics ou privés. 


\title{
UMPa: A Multi-objective, multi-level partitioner for communication minimization
}

\author{
Ümit V. Çatalyürek, Mehmet Deveci, Kamer Kaya, and Bora Uçar
}

\begin{abstract}
We propose a directed hypergraph model and a refinement heuristic to distribute communicating tasks among the processing units in a distributed memory setting. The aim is to achieve load balance and minimize the maximum data sent by a processing unit. We also take two other communication metrics into account with a tie-breaking scheme. With this approach, task distributions causing an excessive use of network or a bottleneck processor which participates in almost all of the communication are avoided. We show on a large number of problem instances that our model improves the maximum data sent by a processor up to $34 \%$ for parallel environments with 4, 16,64 and 256 processing units compared to the state of the art which only minimizes the total communication volume.
\end{abstract}

\section{Introduction}

In parallel computing, the problem of distributing communicating tasks among the available processing units is important. To solve this problem, several graph and hypergraph models are proposed $[\mathbf{6}, \mathbf{7}, \mathbf{9}, \mathbf{1 2}, \mathbf{2 0}]$. These models transform the problem at hand to a balanced partitioning problem. The balance restriction on part weights in conventional partitioning corresponds to the load balance in the parallel environment, and the minimized objective function corresponds to the total communication volume between processing units. Both criteria are crucial in practice for obtaining short execution times, using less power, and utilizing the computation and communication resources better.

In addition to the total data transfer, there are other communication metrics investigated before, e.g., total number of messages sent [19], or maximum volume of messages sent and/or received by a processor $[\mathbf{4}, \mathbf{1 9}]$. Even with perfect load balancing and minimized total data transfer, there can be a bottleneck processing unit which participates in most of the data transfers. This can create a problem especially for data intensive applications where reducing the amount of data transferred by the bottleneck processing unit can improve the total execution time significantly.

2010 Mathematics Subject Classification. Primary 05C65, 05C70; Secondary 90C35.

This work was supported in parts by the DOE grant DE-FC02-06ER2775 and by the NSF grants CNS-0643969, OCI-0904809, and OCI-0904802. 
In this work, given a task graph, our main objective is distributing its tasks evenly and minimizing the maximum amount of data sent by a processing unit. Previous studies addressing different communication cost metrics (such as $[\mathbf{4}, \mathbf{1 9}]$ ) work in two phases. In the first phase, the total volume of communication is reduced, and in the second phase the other metrics are addressed. We propose a directed hypergraph model and partition the related hypergraph with a multi-level approach and a novel $K$-way refinement heuristic. While minimizing the primary objective function, our refinement heuristic also takes the maximum data sent and received by a processing unit and the total amount of data transfer into account by employing a tie-breaking scheme. Therefore, our approach is different from the existing studies in that the objective functions are minimized all at the same time.

The organization of the paper is as follows. In Section 2, the background material on graph and hypergraph partitioning is given. Section 2.3 shows the differences of the graph and hypergraph models and describes the proposed directed hypergraph model. In Section 3, we present our multi-level, multi-objective partitioning tool UMPa (pronounced as "Oompa"). Section 4 presents the experimental results, and Section 5 concludes the paper.

\section{Background}

2.1. Hypergraph partitioning. A hypergraph $\mathcal{H}=(\mathcal{V}, \mathcal{N})$ is defined as a set of vertices $\mathcal{V}$ and a set of nets (hyperedges) $\mathcal{N}$ among those vertices. A net $n \in \mathcal{N}$ is a subset of vertices and the vertices in $n$ are called its pins. The number of pins of a net is called the size of it, and the degree of a vertex is equal to the number of nets it is connected to. In this paper, we will use pins $[n]$ and nets $[v]$ to represent the pin set of a net $n$ and the set of nets vertex $v$ is connected to, respectively. Vertices can be associated with weights, denoted with w[.], and nets can be associated with costs, denoted with c[.].

A $K$-way partition of a hypergraph $\mathcal{H}$ is denoted as $\Pi=\left\{\mathcal{V}_{1}, \mathcal{V}_{2}, \ldots, \mathcal{V}_{K}\right\}$ where

- parts are pairwise disjoint, i.e., $\mathcal{V}_{k} \cap \mathcal{V}_{\ell}=\emptyset$ for all $1 \leq k<\ell \leq K$,

- each part $\mathcal{V}_{k}$ is a nonempty subset of $\mathcal{V}$, i.e., $\mathcal{V}_{k} \subseteq \mathcal{V}$ and $\mathcal{V}_{k} \neq \emptyset$ for $1 \leq k \leq K$,

- union of $K$ parts is equal to $\mathcal{V}$, i.e., $\bigcup_{k=1}^{K} \mathcal{V}_{k}=\mathcal{V}$.

Let $W_{k}$ denote the total vertex weight in $\mathcal{V}_{k}$ (i.e., $W_{k}=\sum_{v \in \mathcal{V}_{k}} \mathrm{w}[v]$ ) and $W_{\text {avg }}$ denote the weight of each part when the total vertex weight is equally distributed (i.e., $\left.W_{\text {avg }}=\left(\sum_{v \in \mathcal{V}} \mathrm{w}[v]\right) / K\right)$. If each part $\mathcal{V}_{k} \in \Pi$ satisfies the balance criterion

$$
W_{k} \leq W_{\text {avg }}(1+\varepsilon), \quad \text { for } k=1,2, \ldots, K
$$

we say that $\Pi$ is $\epsilon$-balanced where $\varepsilon$ represents the maximum allowed imbalance ratio.

For a $K$-way partition $\Pi$, a net that has at least one pin (vertex) in a part is said to connect that part. The number of parts connected by a net $n$, i.e., connectivity, is denoted as $\lambda_{n}$. A net $n$ is said to be uncut (internal) if it connects exactly one part (i.e., $\lambda_{n}=1$ ), and cut (external), otherwise (i.e., $\lambda_{n}>1$ ).

The set of external nets of a partition $\Pi$ is denoted as $\mathcal{N}_{E}$. There are various cutsize definitions [16] for hypergraph partitioning. The one that will be used in this work, which is shown to accurately model the total communication volume [7], is called the connectivity metric and defined as: 


$$
\chi(\Pi)=\sum_{n \in \mathcal{N}} \mathrm{c}[n]\left(\lambda_{n}-1\right) .
$$

In this metric, each cut net $n$ contributes $\mathrm{c}[n]\left(\lambda_{n}-1\right)$ to the cutsize. The hypergraph partitioning problem can be defined as the task of finding a balanced partition $\Pi$ with $K$ parts such that $\chi(\Pi)$ is minimized. This problem is also NP-hard [16].

2.2. $K$-way partitioning and multi-level framework. Arguably, the multilevel approach [3] is the most successful heuristic for the hypergraph partitioning problem. Although, it has been first proposed for recursive-bisection based graph partitioning, it also works well for hypergraphs $[\mathbf{2}, \mathbf{5}, \mathbf{7}, \mathbf{1 3}, \mathbf{1 7}]$. In the multi-level approach, a given hypergraph is coarsened to a much smaller one, a partition is obtained on the the smallest hypergraph, and that partition is projected to the original hypergraph. These three phases will be called the coarsening, initial partitioning, and uncoarsening phases, respectively. The coarsening and uncoarsening phases have multiple levels. In a coarsening level, similar vertices are merged to make the hypergraph smaller. In the corresponding uncoarsening level, the merged vertices are split, and the partition of the coarser hypergraph is refined for the finer one.

Most of the multi-level partitioning tools used in practice are based on recursive bisection. In recursive bisection, the multi-level approach is used to partition a given hypergraph into two. Each of these parts is further partitioned into two recursively until $K$ parts are obtained in total. Hence, to partition a hypergraph into $K=2^{k}$, the recursive bisection approach uses $K-1$ coarsening, initial partitioning, and uncoarsening phases.

Several successful clustering heuristics are proposed to coarsen a hypergraph. Although their similarity metrics aim to reduce the cutsize, they cannot find an optimal solution, since the problem is NP-hard. Hence, an optimal partition of the coarser hypergraph may not be optimal for the finer one. To obtain better partitions, iterative-improvement-based heuristics are used to refine the coarser's partition after projecting it to finer. In practice, Kernighan-Lin (KL) [15] and Fiduccia-Mattheyses (FM) [11] based refinement heuristics that depend on vertex swaps and moves between two parts are used.

2.3. Task graph and communication volume metrics. Let $\mathcal{A}=(\mathcal{T}, \mathcal{C})$ be a task graph where $\mathcal{T}$ is the set of tasks to be executed, and $\mathcal{C}$ is the set of communications between pairs of tasks. We assume that the execution time of each task may differ, hence each task $t \in \mathcal{T}$ is associated with an execution time $\operatorname{exec}(t)$. Each task $t_{i} \in \mathcal{T}$ sends a different amount of data data $\left(t_{i}\right)$ to each $t_{j}$ such that $t_{i} t_{j} \in \mathcal{C}$. The communications between tasks may be uni-directional, That is $t_{i} t_{j} \in \mathcal{C}$ does not imply $t_{j} t_{i} \in \mathcal{C}$. In our parallel setting, we assume owner computes rule and hence, each task of $\mathcal{A}$ is executed by the processing unit to which it is assigned. Let $\mathcal{T}_{i}$ be the set of tasks assigned to processing unit $P_{i}$. Since it is desirable to distribute the tasks evenly, the computational load $\sum_{t \in \mathcal{T}_{i}} \operatorname{exec}(t)$ should be almost the same for each $P_{i}$. In addition to that two heavily communicating tasks should be assigned to the same processing unit since less data transfer over the network is needed in this case. The total amount of data transfer throughout the execution of the tasks is called the total communication volume $(\operatorname{tot} V)$. Note that when a task $t \in \mathcal{T}_{i}$ needs to send data to a set of tasks 
in $\mathcal{T}_{j}$, the contribution to tot $V$ is data(t), since it is enough to send $t$ 's data to $P_{j}$ only once.

Although minimizing the total communication volume is important, it is sometimes preferable to reduce other communication metrics [12]. For example, in the context of one-dimensional partitioning of structurally unsymmetric sparse matrices for parallel matrix-vector multiplies, Uçar and Aykanat used a communication hypergraph model to reduce the maximum of number of messages and the maximum amount of data sent and received by a processor [19] (see also [4] and [18] for other communication metrics).

Let $S V[i]$ and $R V[i]$ be the volumes of communication sent and received by $P_{i}$, respectively. Hence, the total communication volume equals to tot $V=\sum_{i} \operatorname{SV}[i]=$ $\sum_{i} \mathrm{RV}[i]$. In addition to tot $V$, we are interested in two other communication metrics: maximum send volume $(\max S V)$, which equals to $\max _{i}(\mathrm{SV}[i])$; and maximum send-receive volume $(\max S R V)$, which is $\max _{i}(\mathrm{SV}[i]+\mathrm{RV}[i])$.

\section{UMPa: A multi-objective partitioning tool for communication minimization}

3.1. Directed hypergraph model. We propose modeling the task graphs with directed hypergraphs. Given a task graph $\mathcal{A}$, we construct the directed hypergraph model $\mathcal{H}=(\mathcal{V}, \mathcal{N})$ as follows. For each task $t_{i} \in \mathcal{T}$, we have a corresponding vertex $v_{i} \in \mathcal{V}$ and a net $n_{i} \in \mathcal{N}$ where pins $\left[n_{i}\right]=\left\{v_{i}\right\} \cup\left\{v_{j} \mid t_{i} t_{j} \in \mathcal{C}\right\}$, $\mathrm{w}\left[v_{i}\right]=\operatorname{exec}\left(t_{i}\right)$, and $\mathrm{c}\left[n_{i}\right]=\operatorname{data}\left(t_{i}\right)$. In this directed hypergraph model, the communication represented by a net $n$ is flowing from its source vertex, which will be denoted as $s(n)$, to the target vertices $\operatorname{pins}[n] \backslash\{s(n)\}$. Given a partition $\Pi$, let $\delta\left(n, \mathcal{V}_{i}\right)=1$ if $n \cap \mathcal{V}_{i} \neq \emptyset$, and 0, otherwise. Then the data sent and received by $P_{i}$ are equal to $\mathrm{SV}[i]=\sum_{n, s(n) \in \mathcal{V}_{i}} \mathrm{c}[n]\left(\lambda_{n}-1\right)$ and $\mathrm{RV}[i]=\sum_{n, s(n) \notin \mathcal{V}_{i}} \mathrm{c}[n] \delta\left(n, \mathcal{V}_{i}\right)$, respectively. Our primary objective is to minimize $\max S V$, the maximum send volume. While doing this, we also take the maximum send-receive volume and the total communication volume into account. The total volume of communication corresponds to the cutsize definition (2.2) as in the standard hypergraph model. In other words, the sense of direction is not important for the total communication volume tot $V$. On the other hand, the directions of the flow is crucial while minimizing $\max S V$ and $\max S R V$.

To optimize its metrics, UMPa follows the multi-level approach. Instead of a recursive bisection, it adopts a direct $K$-way partitioning. Given the hypergraph, UMPa gradually coarsens it, obtains an initial $K$-way partition for the coarsest hypergraph, and projects it into the original one by uncoarsening and refinement steps at each level.

3.2. Multi-level coarsening phase. In this phase, the original hypergraph is gradually coarsened in multiple levels by clustering subsets of vertices at each level. There are two types of clustering algorithms: matching-based ones and agglomerative ones. The matching-based ones put at most two similar vertices in a cluster, whereas the agglomerative ones allow any number of similar vertices. There are various similarity metrics - see for example $[\mathbf{1}, \mathbf{7}, \mathbf{1 4}]$. All these metrics are defined only on two adjacent vertices (one of them can be a vertex cluster). Two vertices are adjacent if they share a net and they can be in the same cluster if the are adjacent. 
In this work, we use an agglomerative algorithm and the absorption clustering metric using pins $[\mathbf{1}, \mathbf{8}]$. For this metric, the similarity between two adjacent vertices $u$ and $v$ is

$$
\sum_{n \in \text { nets }[u] \cap \text { nets }[v]} \frac{c[n]}{|\operatorname{pins}[n]|-1}
$$

This is also the default metric in $\mathrm{PaToH}[8]$, a well-known hypergraph partitioner. In each level $\ell$, we start with a finer hypergraph $\mathcal{H}^{\ell}$ and obtain a coarser one $\mathcal{H}^{\ell+1}$. If $\mathcal{V}_{C} \subset \mathcal{V}^{\ell}$ is a subset of vertices deemed to be clustered, we create the cluster vertex $u \in \mathcal{V}^{\ell+1}$ where nets $[u]=\cup_{v \in \mathcal{V}_{C}}$ nets $[v]$. We also update the pin sets of the nets in nets $[u]$ accordingly.

Since we need the direction, i.e., source vertex information for each net to $\operatorname{minimize} \max S V$ and $\max S R V$, we always store the source vertex of a net $n \in \mathcal{N}$ as the first pin in pins $[n]$. To maintain this information, when a cluster vertex $u$ is formed in the coarsening phase, we put $u$ to the head of $\operatorname{pins}[n]$ for each net $n$ whose source vertex is in the cluster.

3.3. Initial partitioning phase. To obtain an initial partition for the coarsest hypergraph, we use $\mathrm{PaToH}[\mathbf{8}]$, which is proven to produce high quality partitions with respect to total communication volume metric [7]. We execute $\mathrm{PaToH}$ ten times and get the best partition according to the $\max S V$ metric. We have several reasons to use $\mathrm{PaToH}$. First, although our main objective is minimizing $\max S V$, since we also take tot $V$ into account, it is better to start with an initial partition having a good total communication volume. Second, since tot $V$ is the sum of the send volumes of all parts, as we observed in our preliminary experiments, minimizing it may also be good for both $\max S V$ and $\max S R V$. Also, as stated in [2], using recursive bisection and FM-based improvement heuristics for partitioning the coarsest hypergraph is favorable due to small net sizes and high vertex degrees.

3.4. $K$-way refinement of communication volume metrics. In an uncoarsening level, which corresponds to the $\ell$ th coarsening level, we project the partition $\Pi^{\ell+1}$ obtained for $\mathcal{H}^{\ell+1}$ to $\mathcal{H}^{\ell}$. Then, we refine it by using a novel $K$-way refinement heuristic which is described below.

Given a partition $\Pi$, let a vertex be a boundary vertex if it is in the pin set of at least one cutnet. Let $\Lambda(n, p)=\left|\operatorname{pins}[n] \cap \mathcal{V}_{p}\right|$ be the number of pins of net $n$ in part $p$, and part $[u]$ be the current part of $u$. The proposed heuristic runs in multiple passes where in a pass it visits each boundary vertex $u$ and either leaves it in part $[u]$, or moves it to another part according to some move selection policy. Algorithm 1 shows a pass of the proposed refinement heuristic. For each visited boundary vertex $u$ and for each available part $p$ other than part $[u]$, the heuristic computes how the communication metrics are affected when $u$ is moved to $p$. This is accomplished in three steps. First, $u$ is removed from part $[u]$, and the leave gains on the send/receive volumes of the parts are computed (after line 1). Second, $u$ is put into a candidate part $p$ and the arrival losses on the send/receive volumes are computed (after line 2). Last, the maximum send, maximum send-receive, and total volumes are computed for this move (after line 4).

3.4.1. Move selection policy and tie-breaking scheme. Our move selection policy given in Algorithm 2 favors the moves with the maximum gains on $\max S V$ and never allows a move with negative gain on the same metric. To take other metrics 


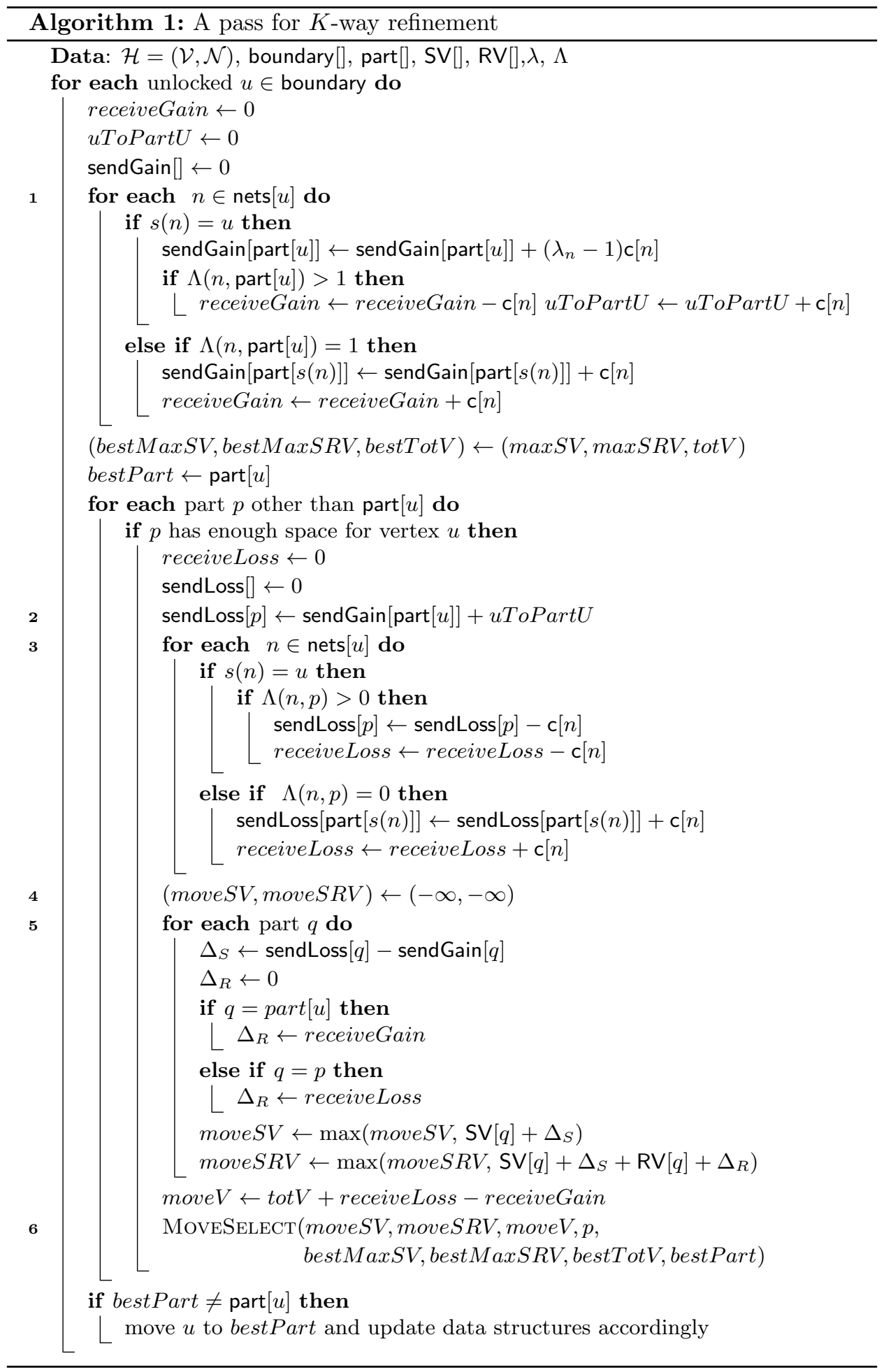


into account, we use a tie-breaking scheme which is enabled when two different moves of a vertex $u$ have the same $\max S V$ gain. In this case, the move with $\max S R V$ gain is selected as the best move. If the gains on $\max S R V$ are also equal then the move with maximum gain on tot $V$ is selected. We do not allow a vertex move without a positive gain on any of the communication metrics. As the experimental results show, this move selection policy and tie-breaking scheme have positive impact on all the metrics.

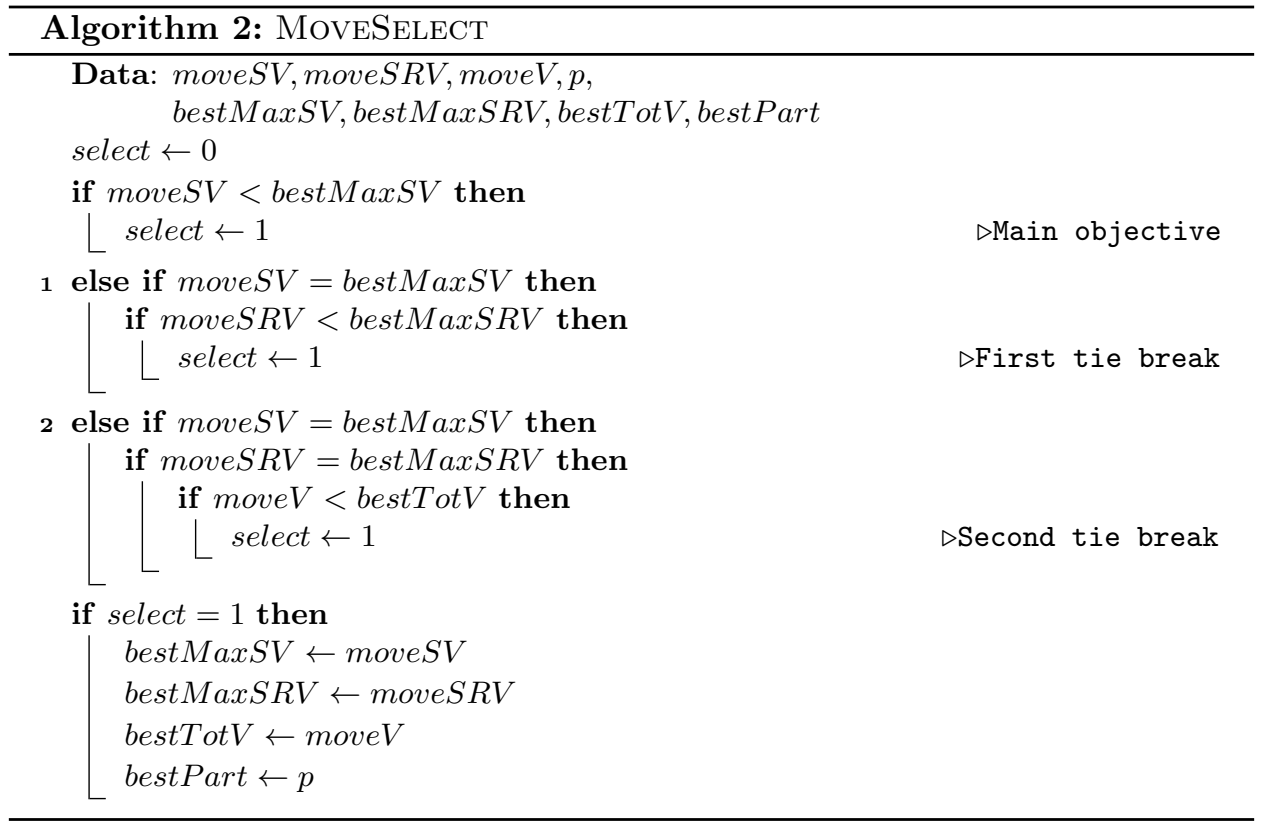

Figure 1 shows a sample graph with 8 vertices and 13 edges partitioned into 3 parts. Assume that this is a partial illustration of boundary vertices, and any move will not violate the balance criteria. Each row in the table contains a possible vertex move and the changes on the communication volume metrics. In the initial configuration, $\max S V=6, \max S R V=9$, and $\operatorname{tot} V=12$. If we move $v_{3}$ from the partition $\mathcal{V}_{2}$ to the partition $\mathcal{V}_{3}$, we reduce all metrics by 1 . On the other hand, if we move $v_{3}$ to $\mathcal{V}_{1}$, we decrease $\max S V$ and $\max S R V$, but tot $V$ does not change. In this case, since its gain on tot $V$ is better, the tie-breaking scheme favors the move $v_{3}$ to $\mathcal{V}_{3}$. Moreover, the moves $v_{4}$ to $\mathcal{V}_{1}, v_{6}$ to $\mathcal{V}_{3}$ and $v_{7}$ to $\mathcal{V}_{3}$ are other move examples where tie-breaking scheme is used. Note that we allow all the moves in the first 13 rows of the table including these two. However, we do not allow the ones in the last three rows.

3.4.2. Implementation details. During the gain computations, the heuristic uses the connectivity information between nets and parts stored in data structures $\lambda$ and $\Lambda$. These structures are constructed after the initial partitioning phase, and then maintained by the uncoarsening phase. Since the connectivity information changes after each vertex move, when a vertex $u$ is moved, we visit the nets of $u$ and update the data structures accordingly. Also, when new vertices become boundary vertices, they are inserted into boundary array and visited in the same pass. 


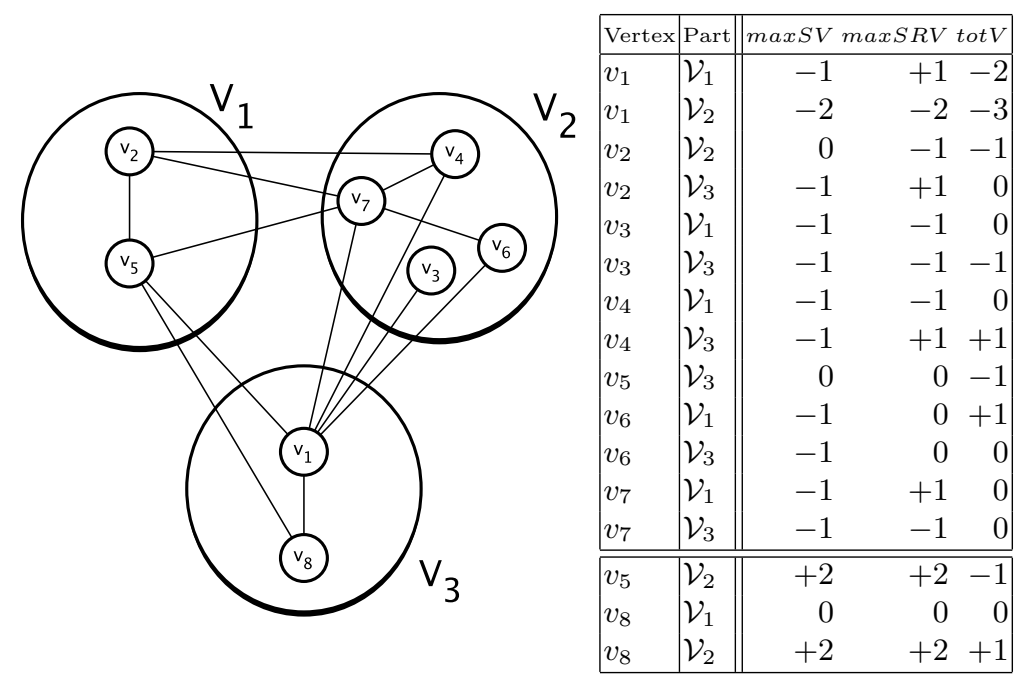

Figure 1. A sample partitioning and some potential moves with their effects on the communication volume metrics. The initial values are $\max S V=6, \max S R V=9$ and $\operatorname{tot} V=12$. A negative value in a column indicates a reduction on the corresponding metric.

If at least one move with a positive gain on $\max S V$ is realized in a refinement pass, the heuristic continues with the next pass. Otherwise, it stops. For efficiency purposes, throughout the execution of a pass, we restrict the number of moves for each vertex $u$. If this number is reached, we lock the vertex and remove it from the boundary. In our experiments, the maximum number of moves per vertex is 4 .

Let $\rho=\sum_{n \in \mathcal{N}}|\operatorname{pins}[n]|$ be the number of pins in a hypergraph. The time complexity of a pass of the proposed refinement heuristic is $\mathcal{O}\left(\rho K+|\mathcal{V}| K^{2}\right)$ due to the gain computation loops at lines 3 and 5 . To store the numbers of pins per part for each net, $\Lambda$, we use a 2-dimensional array. Hence, the space complexity is $\mathcal{O}(K|\mathcal{N}|)$. This can be improved as shown in $[2]$.

\section{Experimental results}

UMPa is tested on a computer with $2.27 \mathrm{GHz}$ dual quad-core Intel Xeon CPUs and 48GB main memory. It is implemented in $\mathrm{C}++$ and compiled with $\mathrm{g}++$ version 4.5.2.

To obtain our data set, we used several graphs from the testbed of 10th DIMACS implementation challenge [10]. We remove relatively small graphs containing less than $10^{4}$ vertices, and also extremely large ones. There are 123 graphs in our data set from 10 graph classes. For each graph, we execute UMPa and other algorithms 10 times. The results in the tables are the averages of these 10 executions.

To see the effect of UMPa's $K$-way partitioning structure and its tie-breaking scheme, we compare it with two different refinement approaches and PaToH. The first approach is partitioning the hypergraph into $K$ with PaToH's recursive bisection scheme and refining it by using the proposed $K$-way refinement algorithm 
TABLE 1. The relative performance of UMPa and PaToH+refinement without tie breaking. The performance are computed with respect to that of $\mathrm{PaToH}$.

\begin{tabular}{|c|c|c|c|c|c|c|c|c|c|}
\hline \multirow[b]{2}{*}{$K$} & \multicolumn{3}{|c|}{$\begin{array}{c}\text { PaToH }+ \text { Refinement } \\
\text { No tie breaking }\end{array}$} & \multicolumn{3}{|c|}{$\begin{array}{c}\text { UMPa } \\
\text { No tie breaking }\end{array}$} & \multicolumn{3}{|c|}{$\begin{array}{c}\text { UMPa } \\
\text { With tie breaking }\end{array}$} \\
\hline & $\max S V$ & $S R V$ & tot $V$ & $\max S V$ & $S R V$ & tot $V$ & $\max S V$ & $S R V$ & tot $V$ \\
\hline 4 & 0.93 & 1.05 & 1.06 & 0.73 & 0.83 & 0.93 & 0.66 & 0.77 & 0.84 \\
\hline 16 & 0.93 & 1.06 & 1.04 & 0.84 & 0.94 & 1.11 & 0.73 & 0.83 & 0.98 \\
\hline 64 & 0.91 & 1.04 & 1.02 & 0.86 & 0.98 & 1.12 & 0.76 & 0.87 & 1.00 \\
\hline 256 & 0.91 & 1.03 & 1.01 & 0.89 & 1.00 & 1.10 & 0.81 & 0.91 & 1.02 \\
\hline Avg. & 0.92 & 1.05 & 1.03 & 0.83 & 0.93 & 1.06 & 0.74 & 0.84 & 0.96 \\
\hline
\end{tabular}

without employing the tie-breaking scheme. The second approach is using UMPa but again without tie breaking. To remove tie breaking, we remove the else statements at lines labeled with 1 and 2 of Algorithm 2.

Table 1 gives the average performance of all these approaches normalized with respect to PaToH's performance. Without tie breaking, refining PaToH's output reduces the maximum send volume by $8 \%$. However, it increases the maximum send-receive and total volumes by $5 \%$ and $3 \%$, respectively. Hence, we do not suggest using the refinement heuristic alone and without tie breaking. On the other hand, if it is used in the multi-level structure of UMPa, we obtain better results even without a tie-breaking scheme.

Table 1 shows that UMPa's multi-level structure helps to obtain $17 \%$ and $7 \%$ less volumes than PaToH's partitions in terms of $\max S V$ and $\max S R V$, respectively. But since PaToH minimizes the total communication volume, there is a $6 \%$ overhead on the tot $V$. Considering $17 \%$ reduction on $\max S V$, this overhead is acceptable. However, we can still reduce all the communication metrics 9\%-to- $10 \%$ more by employing the proposed tie-breaking scheme. For $K=4$, this leads us a $34 \%$ better maximum send volume, which is impressive since even the total communication volume is $16 \%$ less compared with PaToH. Actually, for all $K$ values, UMPa manages to reduce $\max S V$ and $\max S R V$ on the average. The percent of improvement reduces with the increasing $K$. This may be expected since when $K$ is large, the total volume will be distributed into more parts, and the maximum send or send-receive volume will be less. Still, on the average, the reductions on $\max S V, \max S R V$, and tot $V$ are $26 \%, 16 \%$, and $4 \%$, respectively.

Tables 2 and 3 show performance of $\mathrm{PaToH}$ and UMPa in terms of the communication metrics and time. There are 20 graphs in each table selected from 10 graph class in DIMACS testbed. For each graph class, we select the two (displayed consecutively in the tables) for which UMPa obtains the best and worst improvements on $\max S V$. The numbers given in the tables are averages of 10 different executions. For all experiments with $K=16$ parts, as Table 2 shows, UMPa obtains a better $\max S V$ value than $\mathrm{PaToH}$ on the average. When $K=4,64$, and 256, PaToH obtains a better average $\max S V$ only for 16, 4, and 1 graphs, out of 123, respectively.

There are some instances in the tables for which UMPa improves $\max S V$ significantly. For example, for graph ut2010 in Table 2, the $\max S V$ value is reduced from 1506 to 330 with approximately $78 \%$ improvement. Furthermore, for the same graph, the improvements on $\max S R V$ and $\operatorname{tot} V$ are $75 \%$ and $67 \%$, respectively. 
TABLE 2. The maximum send and send-receive volumes, and the total volume for $\mathrm{PaToH}$ and UMPa when $K=16$. The times are given in seconds. There are 20 graphs in the table where two graphs with the best and the worst improvements on $\max S V$ are selected from each class. Each number is the average of 10 different executions.

\begin{tabular}{l||r|r|r|r||r|r|r|r}
\multicolumn{4}{c||}{} & \multicolumn{3}{c||}{ PaToH } & \multicolumn{3}{c}{ UMPa } \\
\cline { 2 - 8 } Graph & maxSV & maxSRV & totV & Time & maxSV & maxSRV & totV & Time \\
\hline coPapersDBLP & 62,174 & 139,600 & 673,302 & 91.45 & 53,619 & 117,907 & 842,954 & 145.47 \\
as-22july06 & 1,506 & 5,063 & 12,956 & 0.63 & 1,144 & 3,986 & 13,162 & 2.70 \\
road_central & 500 & 999 & 3,926 & 112.64 & 279 & 576 & 2,810 & 27.85 \\
smallworld & 12,043 & 24,020 & 188,269 & 3.09 & 10,920 & 21,844 & 174,645 & 19.27 \\
delaunay_n14 & 119 & 235 & 1,500 & 0.19 & 115 & 236 & 1,529 & 0.88 \\
delaunay_n17 & 351 & 706 & 4,100 & 1.09 & 322 & 655 & 4,237 & 2.54 \\
hugetrace-00010 & 2,113 & 4,225 & 25,809 & 93.99 & 2,070 & 4,144 & 28,572 & 43.39 \\
hugetric-00020 & 1,660 & 3,320 & 20,479 & 60.96 & 1,601 & 3,202 & 22,019 & 29.51 \\
venturiLevel3 & 1,774 & 3,548 & 19,020 & 27.41 & 1,640 & 3,282 & 20,394 & 16.01 \\
adaptive & 2,483 & 4,967 & 27,715 & 54.00 & 2,345 & 4,692 & 29,444 & 29.33 \\
rgg_n_2_15_s0 & 146 & 293 & 1,519 & 0.34 & 119 & 254 & 1,492 & 1.03 \\
rgg_n_2_21_s0 & 1,697 & 3,387 & 19,627 & 37.86 & 1,560 & 3,215 & 20,220 & 16.66 \\
tn2010 & 2,010 & 3,666 & 13,473 & 1.26 & 1,684 & 3,895 & 56,780 & 1.54 \\
ut2010 & 1,506 & 2,673 & 3,977 & 0.43 & 330 & 677 & 1,303 & 0.82 \\
af_shell9 & 1,643 & 3,287 & 17,306 & 14.83 & 1,621 & 3,242 & 18,430 & 8.64 \\
audikw1 & 15,119 & 29,280 & 145,976 & 161.23 & 11,900 & 24,182 & 159,640 & 77.16 \\
asia.osm & 63 & 125 & 409 & 40.43 & 30 & 62 & 323 & 7.67 \\
belgium.osm & 141 & 281 & 1,420 & 4.80 & 120.6 & 243 & 1,406 & 1.96 \\
memplus & 986 & 7,138 & 7,958 & 0.23 & 686 & 3,726 & 10,082 & 0.72 \\
t60k & 155 & 310 & 1,792 & 0.29 & 148.5 & 297 & 1,890 & 0.99 \\
\hline
\end{tabular}

When $K=256$ (Table 3) for the graph memplus, UMPa obtains approximately $50 \%$ improvement on $\max S V$ and $\max S R V$. Although tot $V$ increases $26 \%$ at the same time, this is acceptable considering the improvements on the first two metrics.

Table 4 shows the relative performance of UMPa in terms of execution time with respect to $\mathrm{PaToH}$. As expected, due to the complexity of $K$-way refinement heuristic, UMPa is slower than $\mathrm{PaToH}$ especially when the number of parts is large.

\section{Conclusions and future work}

We proposed a directed hypergraph model and a multi-level partitioner UMPa for obtaining good partitions in terms of multiple communication metrics where the maximum amount of data sent by a processing unit is the main objective function to be minimized. UMPa uses a novel $K$-way refinement heuristic employing a tiebreaking scheme to handle multiple communication metrics. We obtain significant improvements on a large number of graphs for all $K$ values.

We are planning to speed up UMPa and the proposed refinement approach by implementing them on modern parallel architectures. We are also planning to investigate partitioning for hierarchical memory systems, such as cluster of multisocket, multi-core machines with accelerators. 
TABLE 3. The maximum send and send-receive volumes, and the total volume for $\mathrm{PaToH}$ and $\mathrm{UMPa}$ when $K=256$. The times are given in seconds. There are 20 graphs in the table where two graphs with the best and the worst improvements on $\max S V$ are selected from each class. Each number is the average of 10 different executions.

\begin{tabular}{l||r|r|r|r||r|r|r|r}
\multicolumn{1}{c||}{} & \multicolumn{7}{c||}{ PaToH } & \multicolumn{4}{c}{ UMPa } \\
\cline { 2 - 8 } Graph & maxSV & maxSRV & totV & Time & maxSV & maxSRV & totV & Time \\
\hline coPapersCiteseer & 7,854 & 16,765 & 577,278 & 224.09 & 5,448 & 11,615 & 579,979 & 658.21 \\
coPapersDBLP & 14,568 & 34,381 & $1,410,966$ & 143.97 & 10,629 & 23,740 & $1,371,425$ & 1038.86 \\
as-22july06 & 1,555 & 7,128 & 28,246 & 1.01 & 617 & 4,543 & 33,347 & 12.62 \\
smallworld & 1,045 & 2,078 & 232,255 & 4.55 & 877 & 1,751 & 208,860 & 36.24 \\
delaunay_n20 & 301 & 600 & 57,089 & 17.98 & 279 & 566 & 58,454 & 68.85 \\
delaunay_n21 & 420 & 844 & 80,603 & 35.01 & 398 & 813 & 83,234 & 107.35 \\
hugetrace-00000 & 407 & 814 & 74,563 & 55.51 & 415 & 831 & 80,176 & 123.66 \\
hugetric-00010 & 502 & 1,004 & 91,318 & 92.45 & 477 & 955 & 97,263 & 167.69 \\
adaptive & 753 & 1,505 & 143,856 & 96.60 & 735 & 1,472 & 152,859 & 224.30 \\
venturiLevel3 & 568 & 1,137 & 107,920 & 49.97 & 564 & 1,132 & 114,119 & 132.02 \\
rgg_n_2_22_s0 & 799 & 1,589 & 145,902 & 151.30 & 724 & 1,495 & 147,331 & 249.23 \\
rgg_n_2_23_s0 & 1,232 & 2,432 & 219,404 & 347.32 & 1,062 & 2,168 & 221,454 & 446.78 \\
ri2010 & 3206 & 5,989 & 281,638 & 0.72 & 2,777 & 5,782 & 279,941 & 8.66 \\
tx2010 & 5,139 & 9,230 & 124,033 & 8.47 & 3,011 & 7,534 & 117,960 & 15.55 \\
af_shell10 & 898 & 1,792 & 174,624 & 89.90 & 885 & 1,769 & 184,330 & 158.04 \\
audikw1 & 4,318 & 8,299 & 680,590 & 322.57 & 3,865 & 7,607 & 692,714 & 822.73 \\
asia.osm & 72 & 146 & 4,535 & 72.37 & 66 & 135 & 4,484 & 18.79 \\
great-britain.osm & 104 & 209 & 11,829 & 50.52 & 82 & 168 & 11,797 & 25.51 \\
finan512 & 199 & 420 & 36,023 & 2.75 & 192 & 437 & 36,827 & 27.70 \\
memplus & 1,860 & 7,982 & 15,785 & 0.49 & 946 & 4,318 & 19,945 & 8.25 \\
\hline
\end{tabular}

TABLE 4. The relative performance of UMPa with respect to Pa$\mathrm{ToH}$ in terms of execution time. The numbers are computed by using the results of 10 executions for each of the 123 graphs in our data set.

\begin{tabular}{l||rrrr||r}
$K$ & 4 & 16 & 64 & 256 & Avg. \\
\hline Relative time & 1.02 & 1.29 & 2.01 & 5.76 & 1.98
\end{tabular}

\section{References}

1. C. J. Alpert and A. B. Kahng, Recent directions in netlist partitioning: A survey, VLSI Journal 19 (1995), no. 1-2, 1-81.

2. Cevdet Aykanat, B. Barla Cambazoglu, and Bora Uçar, Multi-level direct k-way hypergraph partitioning with multiple constraints and fixed vertices, Journal of Parallel and Distributed Computing 68 (2008), no. 5, 609-625.

3. S. T. Barnhard and H. D. Simon, Fast multilevel implementation of recursive spectral bisection for partitioning unstructured problems, Concurrency: Practice and Experience 6 (1994), no. 2, 67-95.

4. R. H. Bisseling and W. Meesen, Communication balancing in parallel sparse matrix-vector multiplication, Electronic Transactions on Numerical Analysis 21 (2005), 47-65.

5. T. N. Bui and C. Jones, A heuristic for reducing fill-in sparse matrix factorization, Proc. 6th SIAM Conf. Parallel Processing for Scientific Computing, SIAM, 1993, pp. 445-452. 
6. Ü. V. Çatalyürek and C. Aykanat, A hypergraph model for mapping repeated sparse matrixvector product computations onto multicomputers, Proc. International Conference on High Performance Computing, December 1995.

7. __ Hypergraph-partitioning based decomposition for parallel sparse-matrix vector multiplication, IEEE Transactions on Parallel and Distributed Systems 10 (1999), no. 7, 673-693.

8. Ü. V. Çatalyürek and C. Aykanat, Patoh: A multilevel hypergraph partitioning tool, version 3.0, Bilkent University, Department of Computer Engineering, Ankara, 06533 Turkey. PaToH is available at http://bmi.osu.edu/ umit/software.htm, 1999.

9. Ü. V. Çatalyürek, Cevdet Aykanat, and Bora Uçar, On two-dimensional sparse matrix partitioning: Models, methods, and a recipe, SIAM Journal on Scientific Computing 32 (2010), no. $2,656-683$.

10. 10th DIMACS implementation challenge: Graph partitioning and graph clustering, 2011, http://www.cc.gatech.edu/dimacs10/.

11. C. M. Fiduccia and R. M. Mattheyses, A linear-time heuristic for improving network partitions, Proc. 19th Design Automation Conference, 1982, pp. 175-181.

12. Bruce Hendrickson and Tamara G. Kolda, Graph partitioning models for parallel computing, Parallel Computing 26 (2000), 1519-1534.

13. Bruce Hendrickson and Robert Leland, A multilevel algorithm for partitioning graphs, Proc. Supercomputing (New York, NY, USA), ACM, 1995.

14. G. Karypis, Multilevel hypergraph partitioning, Tech. Report 02-25, University of Minnesota, Department of Computer Science/Army HPC Research Center, Minneapolis, MN 55455, 2002.

15. B. W. Kernighan and S. Lin, An efficient heuristic procedure for partitioning graphs, The Bell System Technical Journal 49 (1970), no. 2, 291-307.

16. T. Lengauer, Combinatorial algorithms for integrated circuit layout, Wiley-Teubner, Chichester, U.K., 1990.

17. Aleksandar Trifunovic and William Knottenbelt, Parkway 2.0: A parallel multilevel hypergraph partitioning tool, Proc. ISCIS, LNCS, vol. 3280, Springer Berlin / Heidelberg, 2004, pp. 789-800.

18. Bora Uçar and Cevdet Aykanat, Minimizing communication cost in fine-grain partitioning of sparse matrices, Computer and Information Sciences - ISCIS 2003 (A. Yazici and C. Şener, eds.), Lecture Notes in Computer Science, vol. 2869, Springer Berlin / Heidelberg, 2003, pp. 926-933.

19. Bora Uçar and Cevdet Aykanat, Encapsulating multiple communication-cost metrics in partitioning sparse rectangular matrices for parallel matrix-vector multiplies, SIAM J. Sci. Comput. 25 (2004), 1837-1859.

20. C. Walshaw, M. G. Everett, and M. Cross, Parallel dynamic graph partitioning for adaptive unstructured meshes, Journal of Parallel Distributed Computing 47 (1997), 102-108. 


\section{Appendix A. DIMACS Challenge Results}

TABLe 5. The best maximum send volumes for UMPa for the challenge instances. X means UMPa failed to obtain a partition with the desired imbalance value.

\begin{tabular}{l|r|r|r|r|r|r|r|r|r|r} 
& \multicolumn{7}{c}{ Parts } \\
\cline { 2 - 9 } Graph & 2 & 4 & 8 & 16 & 32 & 64 & 128 & 256 & 512 & 1,024 \\
\hline \hline as365 & & & & & & 1,080 & 790 & 590 & 421 & 316 \\
asia.osm & & & & & & 41 & 46 & 60 & 92 & 93 \\
auto & & & & & 2,044 & 1,497 & 1,070 & 733 & 501 \\
coauthorsciteseer & & 10,066 & 7,773 & 5,313 & 3,216 & 2,006 & & & & \\
delaunay_n15 & & & 189 & 154 & 121 & 90 & 70 & & & \\
er-fact1.5-scale23 & & & & $5,707,503$ & $3,933,216$ & $2,091,986$ & $1,154,276$ & 622,913 & & \\
g3_circuit & 1,266 & 1,630 & & & 1,151 & 938 & & 536 & & 58 \\
great-britain.osm & & & & & 134 & 114 & 92 & 78 & & 58 \\
hugebubbles-00010 & & 3,012 & & & 1,948 & 1,522 & & 822 & 609 & \\
hugetric-00000 & 1,274 & 2,206 & & & 1,117 & 804 & & 458 & & \\
kkt_power & & & & 6,162 & 6,069 & 4,508 & & 3,078 & 2,088 & \\
kron_g500-logn17 & 36,656 & 53,381 & 55,314 & 49,657 & 42,272 & & & & \\
kron_g500-logn21 & & & & & & 459,454 & 351,785 & 245,355 & 168,870 & X \\
m6 & 1,487 & & 2,034 & & 1,427 & & 762 & 568 & & \\
nlpkkt160 & & 71,708 & 55,235 & 49,700 & 36,483 & 25,107 & & & \\
nlr & & 2,380 & & 1,563 & & 847 & 623 & 447 & \\
rgg_n_2_18_s0 & & & 516 & 431 & 330 & 248 & 195 & & & \\
\hline
\end{tabular}

The Ohio State University, Dept. of Biomedical Informatics,

Email address: umit@bmi.osu.edu

The Ohio State University, Dept. of Biomedical Informatics,

Email address: mdeveci@bmi.osu.edu

The Ohio State University, Dept. of Biomedical Informatics,

Email address: kamer@bmi.osu.edu

LIP, ENS LyON, LyON 69364, FRANCE,

Email address: bora.ucar@ens-lyon.fr 